\title{
ReaR
}

LECTURA CRÍTICA DE ARTÍCULO

Abril 2020

\section{Técnicas continuas de depuración extrarrenal. ¿Precoces o tardías? ¿Cuál es el momento idóneo para su inicio?}

Artículo original: Barbar SD, Clere-Jehl R, Bourredjem A, Hernu R, Montini F, Bruyère $R$, et al. Timing of Renal-Replacement Therapy in Patients with Acute Kidney Injury and Sepsis. N Engl J Med. 2018 Oct 11;379(15):1431-1442. doi: 10.1056/NEJMoa1803213. ( $\underline{\text { PubMed) (HTML) (PDF) }}$ San Antonio Gil, Á; Hermoso Martínez, J.I.; Redondo Enríquez, J.M.; Martín González-Haba, M.P.

Complejo hospitalario de Cáceres.

\section{Resumen}

El desarrollo de insuficiencia renal aguda (IRA) constituye una problemática frecuente en las unidades de cuidados críticos (UCI), concretamente en las subpoblaciones ingresadas con diagnóstico de sepsis o shock séptico.

En la literatura, las indicaciones de aplicación de TCRR están claras (tanto de origen renal como extrarrenal).

Sin embargo, no parece claro en ningún estudio publicado previamente el momento ideal del inicio de dichas técnicas, ni la repercusión que esto tiene en la morbimortalidad.

El objetivo de este ensayo clínico es analizar si existen diferencias en la mortalidad entre 2 grupos de pacientes con lesión renal aguda y shock séptico, según el inicio precoz o tardío de las TCRR. Es abierto (no hay enmascaramiento), pudiendo caer en sesgo de medición durante la medición de los datos.

Los grupos de estudio fueron homogéneos, con aleatorización al azar. Sin embargo, no especifican el tipo de modalidad de TCRR utilizada.

El tamaño muestral calculado inicialmente según la potencia conferida al estudio no fue alcanzado finalmente.

Las mediciones fueron objetivas. Sin embargo, no aclaran por qué designan como precoz al inicio de las TCRR en las primeras 12 horas desde el desarrollo de LRA y tardío a 48 horas después.

Resultados: No hay diferencias de mortalidad a los 90 días $(\mathrm{P}=0.38$, no significativo). Sin embargo, en el grupo tardío un 38\% no recibieron TCRR, y $17 \%$ lo recibieron precozmente. El grupo tardío presentó de forma significativa menos días con TCRR. No hubo diferencias en días de ventilación mecánica, vasopresores ni estancia en UCI.

\section{Introducción}

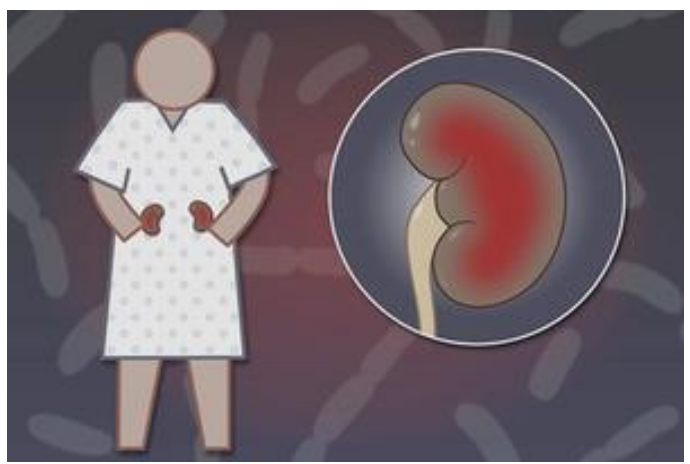

El desarrollo de insuficiencia renal aguda (IRA) constituye una problemática frecuente en las unidades de cuidados críticos (UCI), concretamente en las subpoblaciones ingresadas con diagnóstico de sepsis o shock séptico.

En la literatura, las indicaciones de aplicación de TCRR están claras (tanto de origen renal como extrarrenal).

Sin embargo, no parece claro en ningún estudio publicado previamente el 
momento ideal del inicio de dichas técnicas, ni la repercusión que esto tiene en la morbimortalidad.

El objetivo de este ensayo clínico es analizar si existen diferencias en la mortalidad entre 2 grupos de pacientes con lesión renal aguda y shock séptico, según el inicio precoz o tardío de las TCRR. Es abierto (no hay enmascaramiento), pudiendo caer en sesgo de medición durante la medición de los datos.

Los grupos de estudio fueron homogéneos, con aleatorización al azar. Sin embargo, no especifican el tipo de modalidad de TCRR utilizada.

El tamaño muestral calculado inicialmente según la potencia conferida al estudio no fue alcanzado finalmente.

Las mediciones fueron objetivas. Sin embargo, no aclaran por qué designan como precoz al inicio de las TCRR en las primeras 12 horas desde el desarrollo de LRA y tardío a 48 horas después.

Resultados: No hay diferencias de mortalidad a los 90 días $(\mathrm{P}=0.38$, no significativo). Sin embargo, en el grupo tardío un $38 \%$ no recibieron TCRR, y $17 \%$ lo recibieron precozmente. El grupo tardío presentó de forma significativa menos días con TCRR. No hubo diferencias en días de ventilación mecánica, vasopresores ni estancia en UCI.

Existe una importante asociación entre la sepsis y el desarrollo de lesión renal aguda (LRA) en las UCI (1). Dicha LRA tiene una fisiopatología específica, y una respuesta a las TCRR que puede ser diferente respecto a pacientes con LRA y sin sepsis (2).

El comienzo de estas técnicas debe ser lo más precoz posible en aquellos casos donde surgen complicaciones como hiperpotasemia o acidosis metabólica refractaria al tratamiento. Sin embargo, en ausencia de las mismas, no existen datos suficientes actualmente para apoyar una prescripción precoz o tardía con los estudios publicados hasta la fecha, sin establecerse un claro beneficio de su administración temprana y los posibles riesgos asociados a un comienzo demorado $(3,4)$. Además, las TCRR no están exentas de riesgos.

\section{Descripción del artículo}

Se trata de un ensayo clínico controlado, aleatorizado, abierto, multicéntrico y secuencial, con 2 análisis intermedios.

Su puntuación en la escala de Jadad es de 3(debido a la ausencia de enmascaramiento).

La variable principal consiste en observar si existen diferencias significativas en la mortalidad por cualquier causa a los 90 días en relación al inicio precoz (primeras 12 horas) o tardío (tras 48 horas) de técnicas continuas de reemplazo renal (TCRR) en pacientes con shock séptico y lesión renal aguda (LRA).

Aleatorización centralizada y utilizando una técnica de bloques con minimización.

Tamaño muestral 488 (246 temprano y 242 tardíos). El tamaño muestral estimado según la incidencia de la hipótesis y del error alfa era de 864 (potencia del $80 \%$ y error alfa 0.05 ). Los criterios de inclusión fueron pacientes mayores de 18 años, ingresados en las primeras 48 horas desde el diagnóstico de shock séptico o inicio de drogas vasoactivas, que tuvieran en ese momento LRA según los criterios RIFLE (determinando LRA por alcanzar el estado de fallo en dicha 
clasificación, que englobaba el desarrollo de oliguria $>24$ horas, anuria $>12$ horas, aumento de creatinina 3 veces el nivel basal).

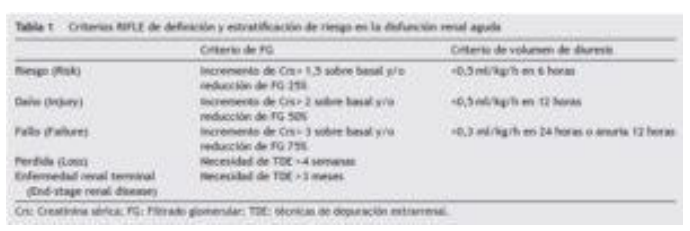

Criterios RIFLE (Revista Med. Intensiva. 2012; vol. 36).

Primero, llevan a cabo un análisis por intención de tratar, utilizando el test de Chi cuadrado para variables cualitativas expresadas como porcentaje. Después, realizan una regresión logística tras ajuste de las covariables, y en un análisis secundario, se evaluó la proporción de muertes a los 28 días y a los 180 días y se trazaron las probabilidades de supervivencia correspondientes mediante las curvas de Kaplan Meier.

Resultados: No existen diferencias de mortalidad a los 90 días entre un inicio precoz o tardío de las TCRR en los pacientes sépticos que desarrollaron LRA $(\mathrm{P}=0.38)$ Obtienen una mortalidad del $58 \%$ en el grupo temprano, y del $54 \%$ en el grupo tardío. El grupo tardío presentó de forma significativa menos días con TCRR (4 versus 2 días, $\mathrm{p}<0.001)$ No hubo diferencias en días de ventilación mecánica, vasopresores ni estancia en UCI.

Conclusiones: No hubo diferencias significativas en la mortalidad a los 90 días tras la asignación a una estrategia temprana para el inicio de la terapia de reemplazo renal y los que fueron asignados a una estrategia retrasada. No se pueden obtener conclusiones debido a la interrupción del estudio por no haber alcanzado el tamaño muestral necesario y no conseguir la potencia suficiente para sacar ninguna conclusión sobre la variable principal, mortalidad a los 90 días.

\section{Análisis Crítico}

Estamos ante un ensayo clínico aleatorizado y multicéntrico publicado en la revista NEJM (con un factor de impacto de 79.258).

Es abierto (no hay enmascaramiento), pudiendo caer en sesgo de información durante la interpretación de los datos. Las características basales de ambos grupos no presentaron diferencias significativas.

Los grupos de estudio fueron aleatorizados al azar 1:1 mediante software informático, creando de esta forma 2 grupos de estudio homogéneos. No queda claro en el texto que tipo de modalidad de TCRR recibieron los pacientes, si hubo diferencias entre ambos grupos.

Las variables que midieron fueron objetivas. Sin embargo, el criterio para designar al inicio de TCRR como temprano (menos de 12 horas desde del diagnóstico de LRA) y tardío (mayor de 48 horas) parece arbitrario. Intervalos de confianza al 95\%, y error alfa establecido en 0,05 .

Los resultados no parecen ser debidos al azar, ya que están reportando una falta de asociación entre un inicio precoz de TCRR y un descenso en la mortalidad en la población evaluada. Sin embargo, esta ausencia de significación puede deberse a la baja potencia del estudio (error de tipo 2). El tamaño muestral calculado inicialmente para conferir la potencia deseada al ensayo no fue logrado finalmente.

Los métodos de medida fueron similares en ambos grupos. Sin embargo, en el grupo tardío, un alto porcentaje de pacientes $(38 \%)$ no 
recibieron TCRR, con lo que queda desequilibrado el tamaño de los grupos comparados.

Los 2 conjuntos son representativos de los pacientes ingresados habitualmente en UCI. Sus características basales (edad, sexo, comorbilidad, puntuación en scores de fallo orgánico) los hacen comparables. Sin embargo, debido a las numerosas pérdidas entre los candidatos a incluirse y los que finalmente se aleatorizan, puede generar un compromiso en la validez externa del estudio.

\section{Conclusiones}

A pesar de ser un ensayo clínico aleatorizado y multicéntrico, publicado en una revista de gran factor de impacto con un importante tamaño muestral, presenta ciertas limitaciones, fundamentalmente en lo relevante al diseño del estudio, debido a la prematura interrupción del mismo, afectando de esta forma a la capacidad de detectar diferencias significativas en relación a la variable principal.

No podemos realizar aseveraciones acerca de si el inicio precoz o tardío de las TCRR en pacientes con shock séptico y LRA repercuten en la morbimortalidad a largo plazo.

\section{Bibliografía}

1. Hoste EA, Bagshaw SM, Bellomo R,Cely CM, Colman R, Cruz DN, et al. Epidemiology of acute kidney injury in critically ill patients: the multinational AKI-EPI study. Intensive Care Med 2015; 41:1411-23 (ubMed)

2. Bellomo R, Kellum JA, Ronco C, Wald R, Martensson J, Maiden M, et al. Acute kidney injury in sepsis. Intensive Care Med 2017; 43:816-28 (PubMed)

3. Gaudry S, Hajage D, Schortgen F, et al. Initiation strategies for renalreplacement therapy in the intensive care unit. $\mathrm{N}$ Engl J Med 2016; 375:122-33 (EJM)

4. Zarbock A, Kellum JA, Schmidt C, Van Aken H, Wempe C, Pavenstädt H et al. Effect of early vs delayed initiation of renal replacement therapy on mortality in critically ill patients with acute kidney injury: the ELAIN randomized clinical trial. JAMA 2016; 315:2190-9 (PubMed)

Correspondencia al autor

Álvaro San Antonio Gil

a.sanantonio.gil@gmail.com

MIR anestesiología y reanimación.

Complejo hospitalario de Cáceres

JM Redondo Enríquez

FEA anestesiología y reanimación.

Complejo hospitalario de Cáceres

Aceptado para el blog en noviembre de 2019 